\title{
Research on Design and Method to Predict Fatigue Life of an Anti-Vibration Mount
}

\author{
Sangeetha N, Mohanraj K
}

\begin{abstract}
In Industry almost all the machinery are subjected to noise, shocks and vibrations when machines are working. These vibrations leads to more frequent repairs and replacements of machine parts also reduce their life span. Antivibration mount is used as a Vibration Control Solutions for machineries. This work is more focused on the importance of anti-vibration mount, which can be used for various mechanical system. This study includes the design of mounts for various functional requirements and fatigue life prediction methods. There are several approaches to predict the fatigue life of mount. Initially, different types of failures in anti-vibration mounts are discussed in detail. Analytical method, Finite Element Method and Experimental approach to predict the fatigue life are analyzed. The strain life approach is considered, incorporate with material properties of mount and another approaches were discussed that are harmonic response, crack nucleation and crack growth mechanics. It is conclude with, the strain life approach is convenient method to predict the fatigue life of antivibration mount, because it give highly non-linear effect to find the critical region of mount.
\end{abstract}

Keywords - Anti-vibration mount, strain life approach, harmonic response, miner's linear method, FEA.

\section{INTRODUCTION}

Vibration mounts can be found in automobiles and power plants, specifically in engine and gearbox. The power train of gear box is mounted on the main frame with the help of anti-vibration bushings (or) mount as shown in fig 1. Bushes are broadly reduces the vibration transmission into the main frame structure and providing long service life. It protect the system from shock load and noise due to torque load. Antivibration mounts are made in two materials, one is the metal and another is natural rubber alternately like sandwich. The natural rubber play vital role to arrest vibration and noise, because it has large deformation and nonlinear property.

Bushes are clamped using square block (or) Plummer block with the static shaft which is connected by arm from main shaft of the gear box. Then it is connected to the main frame using fasteners. The torque load path from rotating turbine to either side of static shaft via main shaft of the gear box and transmit to the anti-vibration mount. Morten Haastrup, illustrate the torque load on bushings using dynamo meter in experimental method and also investigate the displacement and modelling of three type of bushings that are linear, nonlinear and hydro dynamic mounting systems. This paper also show the location of mount and explain about torque load producing for wind turbine using dynamic method [1].

Revised Manuscript Received on August 14, 2019.

Sangeetha N, Faculty, Dept. of Mechanical Engineering, Kumaraguru College of Technology, Coimbatore, Tamil Nadu, India. (E-mail: sangeetha.n.mec@kct.ac.in)

Mohanraj K, Student, Dept. of Mechanical Engineering, Kumaraguru College of Technology, Coimbatore, Tamil Nadu, India.
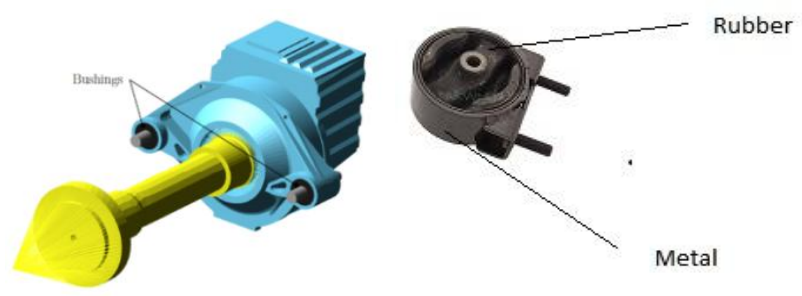

Fig.1 Anti-vibration mounts [2]

\subsection{DESIGN CONSIDERATION FOR ANTI-VIBRATION MOUNT}

It is most important to accommodate the mounting system. Selection and design consideration is based on following information:

- Location of mounting

- Maximum torque load, center of gravity, static weight

- Dimension of unit

- Damping character of mount

In mounting of location there should be balance of weight (or) equally distributed load into mount with the centre of gravity. According to the mounting of location an author discuss the design consideration in engine mounting, to reduce the vibration in the power plant of vehicle. Also deal with the center location engine is calculated by moment of inertia of engine system. In inclined and parallel position mount, elastic center and torque axis is also calculated. Because of this mount configuration, the elastic center will fall on the torque axis [3].

\subsection{FAILURE IN ANTI-VIBRATION MOUNT}

Generally failure occur in engine mounting system due to dynamic character of vehicle or other mechanical system. There is various information to failure occur in mount. When the mount fails due Excess Vibration, it'll transmit more vibration to the rest of the car. This is typical of hydraulic mounts and active mounts, but it can happen to rubber mounts, too. Broken engine mount due to Excess Movement, won't let the engine fall out of your car. Still, it could cause damage to other parts, like coolant hoses, exhaust pipes, or a wiring harness. Clunking Sounds are a good indicator that the engine is loose. This usually occurs on hard acceleration or when engine braking.

Byron Davis, discuss the reason for failure in rotating systems. Various failure mode is explain according to selection and installation of isolators. Then the failure is prevented by database from isolators such as design consideration, increase the damping and stiffness by frequency testing methods [4]. 


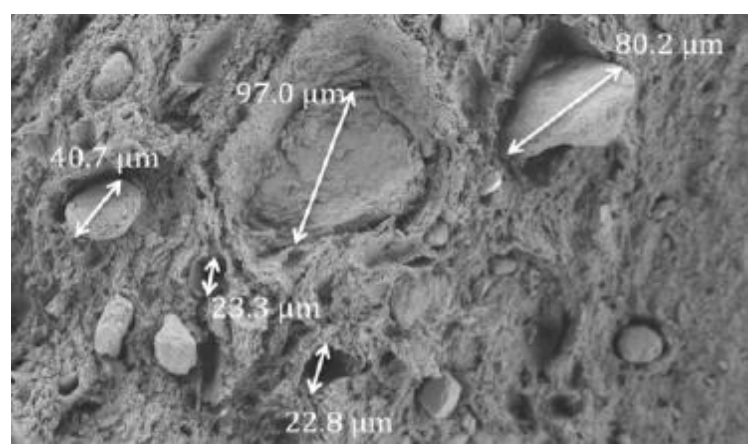

Fig.2 SEM image of fracture surface

Styrene butadiene rubber mount failure is investigated and studied by monotonic and fatigue loading. The rubber mount were used in tracked vehicles which is road wheel backer pads. In monotonic load tension and compression test were performed using ASTM standard specimen. Then the fatigue load is performed by servo-hydraulic load frame and displacement is observed in extensometer. These test were made in room temperature. The behaviour is determined incorporate with strain life approach. The result show that the stiffened with increasing strain and fracture surface observed using scanning electron microscopy shown in fig. 2. [5].

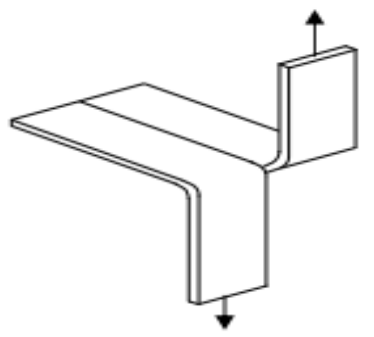

Fig.3 Trouser test specimen [6]

Asare, deals with the problem of crack growth in elastomer is illustrated using theory of Revlin and Thomas (1953). Then extended into Griffith approach. Therefore the energy required to drive the crack at particular rate and is defined as a strain energy release rate. It also called tearing energy. An analytical expression for strain energy release rate is derived from test piece geometries shown in fig.3 [6].Further in carbon the carbon back filled natural rubber. Fatigue damage is investigated under multi-axial and uniaxial loading condition. It is observed in macroscopic and microscopic scale correspond to internal and external crack, then separate in five elementary type. Damage is distinguished by crack growth and crack initiation. This method is used for predict the fatigue crack in location and orientation of damage [7].

\section{ANALYTICAL APPROACH}

To specify the deformation and critical region of rubber component, different type of parameters were discussed such as maximum principal elastic strain, green Lagrangian strain and miners linear method. Incorporate with finite

\subsection{MOONEY REVLIN PARAMETER} element analysis.

By using strain energy theory, the rubber material component deformation is specified in research papers. Revlin, explain and derive the strain-energy function for an in compressible isotropic elastic material must be expressible as a function of two strain invariant. The similar function is discussed Ogden and neo-hooker. Strain function of elastic material is defined by stretch ratio of cross sectional area and length of component. It is used for specify the maximum deformation [8].

\subsection{STRAIN LIFE METHOD}

Babansuryatah, estimate the fatigue life of railway elastomeric pad under compression load. The life time is predicted by material property of rubber using ASTM standard specimen shown in fig.5. The damage parameter is maximum principal elastic strain to find the critical region of pad using FEA. Then the value of strain and material constant of natural rubber is substituted into equation of natural rubber. The predicted life is inversely proportional to strain value that is the increase the strain value is decrease the fatigue life of pad are indicated in fig.4. [9].

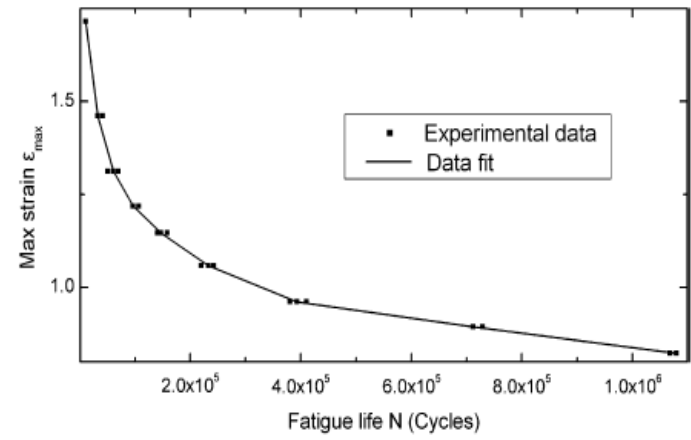

Fig.4 fatigue life prediction of specimen $[10]$

In conduit polymer material, the fatigue life is predicted under various thermal character of rubber using dog-bone rubber specimen in quasi-static loading in quasi-static instron machine. The experimental test is conducted in instron 5582 for uni-axial tensile test. Then the fatigue life predicted using wei-wong unified strain model equation under strain controlled effect in different temperature level [11].Further Natural rubber equation is also used, Touhid Zarrin-ghalami, investigate the fatigue life of cradle mount under constant load amplitude. To define non-linear property of rubber material neo-hooker method is generated incorporate with mooney-revlin parameter. Mars fatemi and natural rubber equation are used to predict the fatigue life of mount. The critical element is defined by maximum strain [7].

Mirza, estimate the fatigue life of rubber component of spherical bearing, in finite element method, crack growth analysis is conducted and tearing energy is predicted using fracture mechanics in experimental test. Then the fatigue life is estimated by size of crack versus life cycles using tearing energy data and fracture mechanics. The loading condition is pitch, roll and longitudinal loading [12]. 


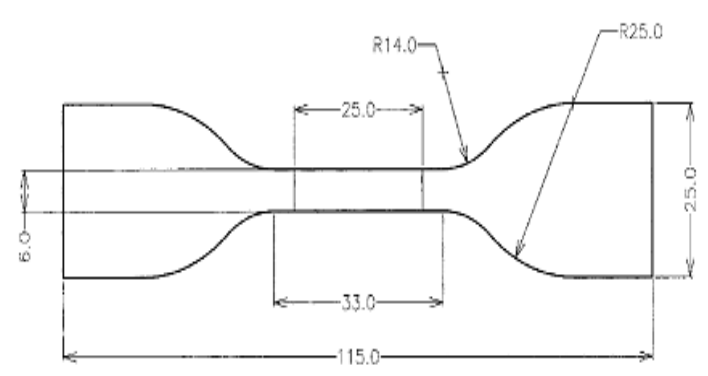

Fig.5 standard specimen of natural rubber [10]

\subsection{FINITE ELEMENT ANALYSIS}

To find the critical region of anti-vibration mount in maximum strain parameter, the finite element analysis is using. Non-linear properties assigned using mooney-revlin parameter for rubber and the component is meshing in four nodded element using ANSYS. Fig.6 illustrate the critical region.

Touhid Zarrin-ghalami, investigate the fatigue life of cradle mount under constant load amplitude. To define nonlinear property of rubber material neo-hooken method is generated incorporate with mooney-revlin parameter. Mars fatemi and natural rubber equation are used to predict the fatigue life of mount. The critical element is defined by maximum strain [7].
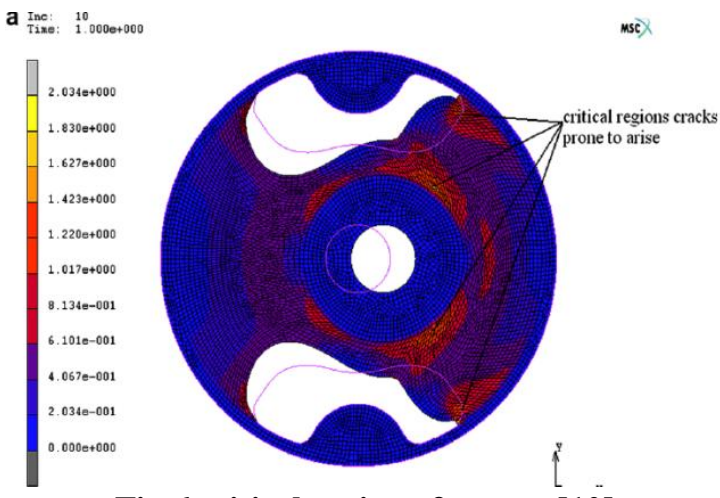

Fig.6 critical region of mount [10]

\subsection{GREEN LAGRANGIAN STRAIN METHOD}

Chung-su woo (2016), develop the fatigue life prediction of automotive rubber component. To estimate the non-linear properties of engine mount, tension, equiv-bi axial and planer test were conducted. In finite element analysis, green Lagrangian strain is a proper damage parameter incorporate with hyper-elastic material model of Mooney-Revlin parameter and Ogden 3parameter. The 3 dimensional dumbbell specimen is used for nonlinear properties of rubber. The green-Lagrangian strain and displacement value is substituted into NR equation. The fatigue life is decrease with increase in displacement [13]

\subsection{MINER'S LINEAR METHOD}

S. Zarrin Ghalami also predict the fatigue life of cradle mount under variable amplitude load. The damage is defined with crack initiation by miner's linear damage rule. Miner's linear rule is defined as ratio of number of load to fatigue initiation life with the damage. The life time is predicted by maximum strain with crack length of mount which is substituted into NR equation [14].

\section{EXPERIMENTAL ANALYSIS \& RESULTS}

There is various experimental methods were discussed depending upon different types of rubber component according to loading condition and boundary condition.

Zine, investigate the fatigue life of styrine butadiene (SBR) rubber under monotonic loading in instron testing device with strain controlled effect. To identify the material constant of rubber, planer tension and uni-axial tension specimen were used. Then the fatigue life is predicted by equation of relationship between material constant and damage parameter. The non-linear behavior is described by Ogden model with 3 parameter [15].Then another method is using estimated the fatigue life under multi-axial loading in two types of vulcanized rubber with various ingredients. Shear strain versus number of cycles and strain amplitude versus number of cycles were predicted by non-proportional loading and torsion-tension loading in load cell. The fatigue fracture surface is also studied in micrography method [16].In another paper fatigue life is investigated along with the temperature measurements in materials like rubber. The life time among heat built-up depending upon protocol and micro-tomography that is, in loading condition the temperature is measured. Then the life time is predicted by Wohler curve in terms of maximum strain and number of cycles [17].

Kyung-Ah Kwon, deals with the synthetic rubber material which is used in brow suspension. The life time is estimated under loading and unloading condition in servo hydraulic machine. Then the life is predicted by force-strain curve. The life time is varying as dissipation of cyclic energy. Also discussed stress relaxation using force versus time and author explain the importance of bubble inflation in fatigue property of elastomer material. Various approaches and fracture mechanics incorporate with strain amplitude and maximum dynamic stored energy under compression loading in UTM machine. The bubble inflation is derived from stretch ratio and strain invariant [18], [19].

The experimental test is conducted on automotive jounce bumper to predict life time in terms of load-displacement and also has explain the Mullin's effect shown in fig.7. It can be explained by stress softening effect, after certain stage of displacement, the stress softening effect will be static condition. A special jig is designed to prevent trapped air to be compressed. Then it is allow the air flow inside the chamber. The fatigue prediction is compared with the literature survey of life time of natural rubber and SBR under strain controlled [2]. 


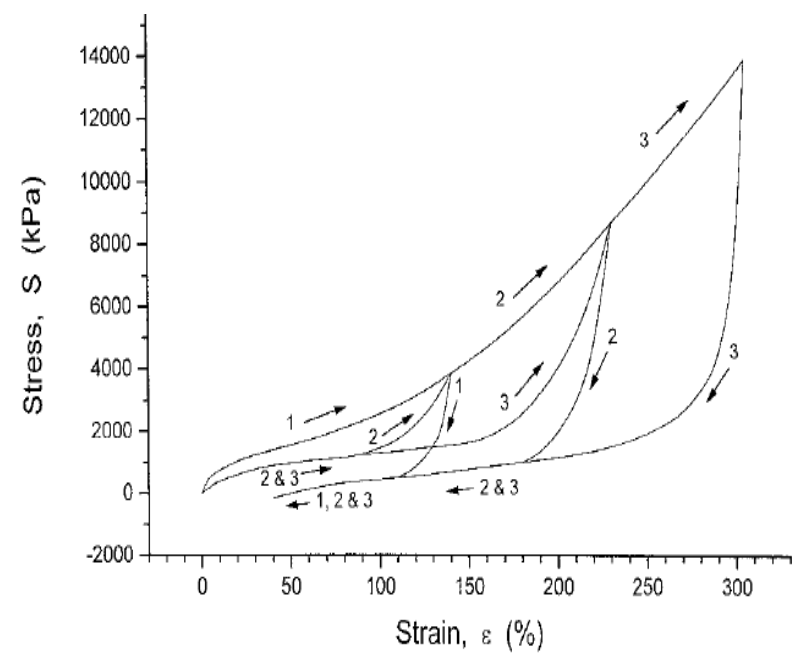

Fig.7 Mullin's effect of natural rubber[12]

Matjaz Mrsnik, estimate the life time using frequency time domain method. Electro dynamic shaker with accelerator is the experimental setup for vibration test. The frequency range is $10-1000 \mathrm{HZ}$. The estimated fatigue life from time domain method is compared with miner's liner method incorporate with S-N curve [6].Also predict the fatigue life of cradle mount under variable amplitude load. The damage is defined with crack initiation by miner's linear damage rule. Miner's linear rule is defined as ratio of number of load to fatigue initiation life with the damage. The life time is predicted by maximum strain with crack length of mount which is substituted into NR equation [15].The frequency is measured using accelerometer. ZoranIlic, investigate the efficiency of vibration absorber in the pilot seat of piston propeller aircraft which is powered by six cylinder engine aircraft. The efficiency is measured using piezo accelerator then the acceleration is evaluated in all direction [20].

Flamm conducted the experimental on carbon back filled rubber material. There is two types of specimen were used, tensile bar and waited bar. The Wohler curve is interpolated under compression and tensile test in the specimens, in terms of strain Lagrangian and tensile test in the specimens. Also explain the crack initiation, crack growth and miners linear method were discussed as fundamental [21].

\section{SUMMARY OF LITERATURE SURVEY:}

In general, vibration is inevitable in various mechanical system because of dynamic motion to get advantage of system, it may prevent by mounting system or methods. It is also provide the accommodation to the system. The material properties of anti-vibration mount play vital role to arrest and isolate the vibration. The following methods and approaches are used to investigate the fatigue life of antivibration mount,

1) For the anti-vibration mount in linear materials like structural steel to find fatigue life of mount, conventional methods is used that is stress or strain life equations of materials.

2) Natural rubber materials are widely used in heavy load system. To estimate the fatigue elastomer with metal, strain life approach is proper damage parameter.
3) Various rubber were used to withstand the load, styrene butadiene rubber, natural rubber with variety of ingredients.

4) To specify the deformation of anti-vibration mount, strain energy theory that is mooney-revlin, neohooker, Ogden material model are using with the natural rubber equation.

5) According to structure, position and materials of mount, various testing methods are used, uni-axial and multi axial.

6) By using FE analysis, maximum principle elastic can be find in the anti-vibration mount. The ratio of maximum and minimum strain value of mount substituted in crack growth and crack nucleation to find the total life of anti-vibration mount.

\section{REFERENCE}

1. Morten Haastrup, Modeling of Wind Turbine Gearbox Mounting. Modeling, Identification and Control, Vol. 32, No. 4, (2011), pp. 141-149, ISSN 1890-1328.

2. M.S.A.Samad, Durability of automotive jounce bumper. Materials and Design 32 (2011) 1001-1005.

3. F. F. Timpner, Design Considerations in Engine Mounting. International Automotive Engineering Congress Detroit, Michigan January (1965) 11-15,

4. J. Byron Davis, Small Deviations and Big Failures in Vibration and Noise Isolation, Sound\& Vibration Magazine/December (2010),14-17.

5. H.R. Brown, Mechanical behaviour of and fatigue studies of rubber components used in tracked vehicles. OMB No. 07040188, 2010, 1-8R.

6. Matjaz Mrsnik Frequency-domain methods for a vibrationfatigue-life estimation - Application to real data. International Journal of Fatigue 47 (2013) 8-17.

7. Touhid Zarrin-ghalami and Ali Fatemi, Fatigue life predictions of rubber components: Applications to an automobile cradle mount. Journal of Automobile Engineering (2013)227(5) 691-703 I Mech E

8. S.Rivlin, The Strain Energy Function for Elastomer transaction of the society of rheology 20:4(1976), 545-557

9. Asare,failure of rubber component under fatigue(2013). Athesis,https:/qmro.qmul.ac.uk/jspui/handle/123456789/8407 ,1-200.

10. Qian Li, Fatigue life prediction of a rubber mount based on test of material properties and FEA, Engineering Failure Analysis 16 (2009) 2304-2310.

11. Pei Chen, Strain-controlled fatigue life and modeling of conduit polymers, Material Science (2011) 46:1902-1912.

12. S. Mirza.Modelling and durability assessment for rubber components in rail vehicles,Plastics, Rubber and Composites (2011) vol 40 no 4, 185-193

13. Chang-Su Woo, Fatigue life prediction methodology of automotive rubber component. The (2016) structures congress(structure 16).1-7

14. T. Zarrin-Ghalami and A. Fatemi. Cumulative fatigue damage and life prediction of elastomeric components. Fatigue Fract Engg Mater Struct00, (2012), 1-10

15. A.Zine.Rubber,fatigue life under multiaxial loading: Numerical and experimental investigations.International Journal of Fatigue 33 (2011) 1360-1368.

16. Y. P. Wang,Microscopic mechanism of multi-axial fatigue of vulcanized natural rubber.Plastics, Rubber and Composites (2011)vol 40 no 10, 491-496. 
17. V.LeSaux, An energetic criterion for the fatigue of rubbers: an approach based on a heat build-up protocol and tomography measurements. Procedia Engineering 2 (2010) 949-958.

18. Stephen Jerrams, The Significance of Equi-biaxial Bubble Inflation in Determining Elastomeric Fatigue Properties.Published in the book Elastomers edited by Anna Boczkowska(2012) (ISBN 979-953-307-1019-5), InTech.

19. Kyung-Ah Kwon, Stress-relaxation and fatigue behaviour of synthetic brow-suspension materials. Journal of the mechanical behavior of bio medical materials 42(2015)116128

20. ZoranIlic, The efficiency of passive vibration damping on the pilot seat of piston propeller aircraft.Measurement 95 (2017) 21-32.

21. M. Flamm,Effects of very high loads on fatigue life of NR elastomer materials.International Journal of Fatigue 33 (2011) 1189-1198 